Dynamic Systems and Applications 30 (2021) No.12, 1792-1801

\title{
UNIQUENESS RESULTS OF FUZZY FRACTIONAL DIFFERENTIAL EQUATIONS UNDER KRASNOSELSKII-KREIN CONDITIONS
}

\author{
YANLI XI ${ }^{1}$, XUPING ZHANG ${ }^{1, *}$, AND YOUHUI SU ${ }^{2, *}$ \\ ${ }^{1}$ Department of Mathematics, Northwest Normal University, Lanzhou \\ 730070, P.R. China \\ ${ }^{2}$ School of Mathematics and Statistics, Xuzhou University of Technology, \\ Xuzhou 221018, P.R. China
}

\begin{abstract}
In this article, we consider a new uniqueness results for a periodic boundary value problem of fuzzy fractional differential equations (FFDES) involving Caputo generalized Hukuhara differentiability with the Krasnoselskii-Krein condition. To this purpose, the equivalent integral forms of FFDEs are determined and then these are used to study the convergence of the Picard successive approximations.
\end{abstract}

AMS (MOS) Subject Classification. 03E72, 34K36, 47S40.

Key Words and Phrases. Fuzzy fractional differential equations, Periodic boundary value problem, Caputo generalized Hukuhara differentiability, Krasnoselskii-Krein condition.

\section{INTRODUCTION}

Fractional differential equation is an important mathematical tool to simulate dynamic system, which can objectively explain and reveal natural phenomena and laws [1]-[4]. Inaccuracies of different types of variables, parameters and data are often encountered when dealing with practical problems. For example, randomness and uncertainty. The theory of stochastic differential equations has been developed for stochastic problems. Therefore, it is necessary to consider the uncertainty factor when dealing with the problem of dynamic system. The random differential equation on real number space is extended to fuzzy number space, that is, fuzzy random differential equation. L. A. Zadeh [5] proposed the fuzzy set theory, which provided an important theoretical basis for dealing with uncertain problems. Since then, fuzzy mathematics has made great progress in both theory and application. In theory, there are important branches of fuzzy analysis, fuzzy topology, fuzzy algebra, etc., and their applications cover artificial intelligence, system control, cluster analysis, pattern recognition, image processing, decision analysis and optimization, etc.

Received September 2, 2021

$\$ 15.00$ (c) Dynamic Publishers, Inc.

www. dynamicpublishers.org;
ISSN 1056-2176(Print); ISSN 2693-5295 (online)

https://doi.org/10.46719/dsa202130.12.04

* Corresponding author. 
Fuzzy differential equations are an effective tool to simulate dynamical systems with uncertainty. By using the Hukuhara derivative ( $H$-derivative) of the fuzzy valued function defined by M. Puri and D. Ralescu [7], O. Kaleva [6] introduced the fuzzy differential equation, and established the existence and uniqueness theory of the solution of the initial value problem. Subsequently, many scholars at home and abroad have conducted in-depth studies on the existence and uniqueness of solutions for differential and integral equations of different types under this framework [8]-[11] . In 1990, J. P. Aubin [12] proposed fuzzy differential inclusion based on the differential inclusion theory of set-valued functions. Recently, the literature [13] used the method of differential inclusion to discuss the periodicity and boundedness of the solutions for first-order linear fuzzy differential equations. Although this method has certain advantages, it lacks the concept of derivative or differentiation of fuzzy value functions under the environment of differential inclusion. Therefore, this research method cannot promote the development of fuzzy value function calculus theory. In order to overcome these shortcomings, B. Bede and S. G. Gal [14] proposed the concept of strong generalized derivative ( $g H$-derivative) with the help of $H$-difference and unilaterally defined derivatives, and studied the initial value problem of fuzzy differential equations.

In 2013, B. Bede and L. Stefanini [15] introduced the generalized $H$-derivative with the help of the generalized $H$-difference ( $g H$-difference) [16] of fuzzy numbers. At the same time, they prove that $g H$-differentiable is equivalent to $g H$-differentiable when the switching point is limited. When dealing with practical problems, the switching points of the functions encountered are usually limited. Therefore, it is still necessary to study fuzzy differential equations under $g H$-differentiable condition. In recent years, many scholars have devoted themselves to the study of the existence and uniqueness of solutions of fuzzy differential equations under $g H$ - derivatives [17]-[22]. E. J. Villamizar-Roa et al. [23] proved the existence and uniqueness of solutions of fuzzy initial value problems under $g H$ - differentiability by means of fixed-point theorems for weakly contractive mappings on posets. R. Alikhani and F.Bahrami [24] proved the existence and uniqueness of global solutions for first order nonlinear fuzzy Integro-differential equations in the case of $g H$ - differentiability by using the method of upper and lower solutions. From the above statement, it can be seen that the current researches on the theory of fuzzy differential equations mainly focus on the existence and uniqueness of the solutions for equations under $g H$-derivative or $g H$-derivative.

As a further work, we consider in this paper the uniqueness of the solution for a class of fuzzy fractional differential equations as follows

$$
\left\{\begin{array}{l}
{ }_{g H}^{C} D_{*}^{\alpha} y(x)=f(x, y(x)), \quad x \in(0, T], \\
y(0)=\lambda y(T)
\end{array}\right.
$$


where $f:[0, T] \times R_{\mathcal{F}} \rightarrow R_{\mathcal{F}}$, is continuous fuzzy-valued function, $\lambda \in(0,1) \cup(1,+\infty)$, and $d(f(x, y(x)), 0) \leq M$ on $E_{0}, E_{0}=\{(x, y(x)): x \in[0, T], d(y(x), y(0)) \leq b\}, M T \leq$ $b$, where $d$ Hausdorff distance.

The purpose of this paper is to introduce the generalized Hukuhara differentiability of fuzzy Caputo fractional differential equation and discuss the krasnoselskii-Krein type unique results of FFDES (1).

\section{THE OPERATOR THEORETIC FORMULATION}

In this section, we briefly introduce some definitions, notations and results related to fuzzy functions, which will be referred to throughout this paper.

The basic definition of fuzzy numbers is given in [21].

We denote the set of all real numbers by $R$ and the set of all fuzzy numbers on $R$ is indicated by $R_{\mathcal{F}}$. A fuzzy number is a mapping $u: R \rightarrow[0,1]$ with the following properties:

(a) $u$ is upper semi-continuous;

(b) $u$ is fuzzy convex, i.e., $u(\lambda x+(1-\lambda) y) \geq \min \{u(x), u(y)\}$ for all $x, y \in R, \lambda \in$ $[0,1]$

(c) $u$ is normal, i.e., $\exists x_{0} \in R$ for which $u\left(x_{0}\right)=1$;

(d) supp $u=\{x \in R \mid u(x)>0\}$ is the support of the $u$, and its closure cl(suppu) is compact.

A fuzzy number function defined on the real set $R$ and valued in $R_{\mathcal{F}}$ is called a fuzzyvalued function, that is, $f: R \rightarrow R_{\mathcal{F}}$. For $r \in(0,1]$ denote $[u]^{r}=\{x \in R \mid u(x) \geq r\}$ and $[u]^{0}=\operatorname{cl}\{x \in R \mid u(x)>0\}$. Then it is well-known that the $r$-level set of $u$, $[u]^{r}=\left[u^{-}(r), u^{+}(r)\right]$, is a closed interval for all $r \in[0,1]$. For $u, v \in R_{\mathcal{F}}$, and $k \in R$, the addition and scalar multiplication are defined by

$$
[u+v]^{r}=[u]^{r}+[v]^{r},[k \cdot u]^{r}=k \cdot[u]^{r}, \forall r \in[0,1]
$$

Let $u, v \in R_{\mathcal{F}}$. If there exists $w \in R_{\mathcal{F}}$ such that $u=v+w$, then $w$ is called the $H$-difference of $u$ and $v$, and it is denoted by $u \ominus v$. In this paper, the sign " $\ominus$ " always stands for $H$-difference, and also note that $u \ominus v \neq u+(-1) v$. The generalized Hukuhara difference of two fuzzy number $u, v \in R_{\mathcal{F}}$ ( $g H$-difference for short) is defined as follows:

$$
u \ominus_{g H} v=w \Leftrightarrow\left\{\begin{array}{l}
(i) u=v+w, \text { or } \\
(i i) v=u+(-1) w .
\end{array}\right.
$$

The Hausdorff distance between fuzzy numbers given by $R_{\mathcal{F}} \times R_{\mathcal{F}} \rightarrow[0,+\infty)$,

$$
d(u, v)=\sup _{r \in[0,1]} \max \left\{\left|u^{-}(r)-v^{-}(r)\right|,\left|u^{+}(r)-v^{+}(r)\right|\right\} .
$$


Then it is easy to see that $d$ is a metric in $R_{\mathcal{F}}$ and the following properties of the metric $d$ are valid(see [25]):

(1) $d(u+w, v+w)=d(u, v), \quad \forall u, v, w \in R_{\mathcal{F}}$

(2) $d(k u, k v)=|k| d(u, v), \quad k \in R, u, v \in R_{\mathcal{F}}$;

(3) $d(u+v, w+z) \leq d(u, w)+d(v, z), \quad \forall u, v, w, z \in R_{\mathcal{F}}$;

(4) $\left(d, R_{\mathcal{F}}\right)$ is a complete metric space.

Let $r$-level representation of fuzzy-valued function $f:[a, b] \rightarrow R_{\mathcal{F}}$ expressed by $f(x ; r)=\left[f^{-}(x ; r), f^{+}(x ; r)\right], x \in[a, b], r \in[0,1]$. Say that the fuzzy-valued function $f$ is integrable on $[a, b]$, if the function $f$ is continuous in the metric $d$, and its definite integral exists, we have

$$
\int_{a}^{b} f(x ; r) d x=\left[\int_{a}^{b} f^{-}(x ; r) d x, \int_{a}^{b} f^{+}(x ; r) d x\right] .
$$

Definition 2.1. ([26],[27]) The generalized Hukuhara derivative of a fuzzy-valued function $f:(a, b) \rightarrow R_{\mathcal{F}}$ at $x_{0}$ is defined as

$$
f_{g H}^{\prime}\left(x_{0}\right)=\lim _{h \rightarrow 0} \frac{f\left(x_{0}+h\right) \ominus_{g H} f\left(x_{0}\right)}{h} .
$$

If $f_{g H}^{\prime}\left(x_{0}\right) \in R_{\mathcal{F}}$, we say that $f$ is generalized Hukuhara differential ( $g H$-differentiable for short) at $x_{0}$. Also, we say that $f$ is $(i)-g H$ differentiable at $x_{0}$, if $f_{g H}^{\prime}\left(x_{0} ; r\right)=$ $\left[f^{\prime}-\left(x_{0} ; r\right), f^{\prime}+\left(x_{0} ; r\right)\right], r \in[0,1]$, and $f$ is $(i i)-g H$ differentiable at $x_{0}$, if $f_{g H}^{\prime}\left(x_{0} ; r\right)=$ $\left[f^{\prime}+\left(x_{0} ; r\right), f^{\prime}-\left(x_{0} ; r\right)\right], r \in[0,1]$.

Definition 2.2. ([28]) Let $f:[a, b] \rightarrow R_{\mathcal{F}}$, the fuzzy Riemann-Liouville integral of fuzzy-valued function $f$ is defined as follows:

$$
\left(\Im_{a}^{\alpha} f\right)(x)=\frac{1}{\Gamma(\alpha)} \int_{a}^{x} \frac{f(t)}{(x-t)^{1-\alpha}} d t, \alpha \in(0,1] .
$$

Definition 2.3. ([29]) Consider $f:[a, b] \rightarrow R_{\mathcal{F}}$, fractional derivative of $f(t)$ in the Caputo sense is defined as

$$
\left({ }^{C} D_{*}^{\alpha} f\right)(x)=\frac{1}{\Gamma(m-\alpha)} \int_{a}^{x}(x-t)^{m-\alpha-1} f^{m}(t) d t, m-1<\alpha<m, m \in N .
$$

Let us denote $C^{R_{\mathcal{F}}}[a, b]$ as the space of all continuous fuzzy-valued functions on $[a, b]$, and we denote the space of all Lebesgue integrable fuzzy-valued functions on interval $[a, b] \subset R$ by $L^{R_{\mathcal{F}}}[a, b]$.

Definition 2.4. Let $f_{g H}^{(m)} \in C^{R_{\mathcal{F}}}[a, b] \cap L^{R_{\mathcal{F}}}[a, b]$. The fuzzy Caputo $g H$-fractional differentiability of fuzzy-valued function $f$ is defined as following

$$
\left({ }_{g H}^{C} D_{*}^{\alpha} f\right)(x)=\Im_{a}^{m-\alpha}\left(f_{g H}^{(m)}\right)(x)=\frac{1}{\Gamma(m-\alpha)} \int_{a}^{x}(x-t)^{m-\alpha-1}\left(f_{g H}^{(m)}\right)(t) d t,
$$

where $m-1<\alpha<m, m \in N, x>a$. 
In this paper, we only consider Caputo $g H$-fractional differentiability of order $0<$ $\alpha \leq 1$ for fuzzy-valued function $f$, so equation (2) can be written as the following form

$$
\left({ }_{g H}^{C} D_{*}^{\alpha} f\right)(x)=\Im_{a}^{1-\alpha}\left(f_{g H}^{\prime}\right)(x)=\frac{1}{\Gamma(1-\alpha)} \int_{a}^{x}(x-t)^{-\alpha}\left(f_{g H}^{\prime}\right)(t) d t .
$$

Definition 2.5. Let $f:[a, b] \rightarrow R_{\mathcal{F}}$ be Caputo $g H$-differentiable at $x_{0} \in(a, b)$. We say that $f$ is ${ }^{C}[(i)-g H]$ differentiable at $x_{0}$, if

$$
\left({ }_{g H}^{C} D_{*}^{\alpha} f\right)\left(x_{0} ; r\right)=\left[\left({ }^{C} D_{*}^{\alpha} f^{-}\right)\left(x_{0} ; r\right),\left({ }^{C} D_{*}^{\alpha} f^{+}\right)\left(x_{0} ; r\right)\right], r \in[0,1],
$$

and $f$ is ${ }^{C}[(i i)-g H]$ differentiable at $x_{0}$, if

$$
\left({ }_{g H}^{C} D_{*}^{\alpha} f\right)\left(x_{0} ; r\right)=\left[\left({ }^{C} D_{*}^{\alpha} f^{+}\right)\left(x_{0} ; r\right),\left({ }^{C} D_{*}^{\alpha} f^{-}\right)\left(x_{0} ; r\right)\right], r \in[0,1] .
$$

where $\left({ }^{C} D_{*}^{\alpha} f^{-}\right)\left(x_{0} ; r\right)=\frac{1}{\Gamma(1-\alpha)} \int_{a}^{x} \frac{{f^{-}}^{\prime}(t ; r)}{(x-t)^{\alpha}} d t,\left({ }^{C} D_{*}^{\alpha} f^{+}\right)\left(x_{0} ; r\right)=\frac{1}{\Gamma(1-\alpha)} \int_{a}^{x} \frac{f^{+^{\prime}}(t ; r)}{(x-t)^{\alpha}} d t$.

Theorem 2.6. Let $\lambda \in(0,1)$ and $\alpha \in(0,1]$, if $y$ is ${ }^{C}[(i)-g H]$ differentiable, then (1) is equivalent to the following integral equation

$$
\begin{aligned}
& y(x) \\
= & \frac{\lambda}{(1-\lambda) \Gamma(\alpha)} \int_{0}^{T}(T-s)^{\alpha-1} f(s, y(s)) d s+\frac{1}{\Gamma(\alpha)} \int_{0}^{x}(x-s)^{\alpha-1} f(s, y(s)) d s, x \in[0, T] .
\end{aligned}
$$

Proof. Let $y(0)=y_{0} \in R_{\mathcal{F}}$. According to [30], if $y$ is ${ }^{C}[(i)-g H]$ differentiable, equation (1) has a differentiable solution

$$
y(x)=y_{0}+\frac{1}{\Gamma(\alpha)} \int_{0}^{x}(x-s)^{\alpha-1} f(s, y(s)) d s, x \in[0, T] .
$$

Since the boundary value condition $y(x)=y_{0}$ is satisfied, then (4) must satisfy

$$
y_{0}=\lambda\left(y_{0}+\frac{1}{\Gamma(\alpha)} \int_{0}^{T}(T-s)^{\alpha-1} f(s, y(s)) d s\right)
$$

we have,

$$
y_{0}=\frac{\lambda}{(1-\lambda) \Gamma(\alpha)} \int_{0}^{T}(T-s)^{\alpha-1} f(s, y(s)) d s .
$$

By (4) and (5), we obtain equation (3).

Theorem 2.7. Let $\lambda \in(1,+\infty)$ and $\alpha \in(0,1]$, if $y$ is ${ }^{C}[(i i)-g H]$ differentiable, then (1) is equivalent to the following integral equation

$$
\begin{aligned}
& y(x) \\
= & \frac{\lambda}{(1-\lambda) \Gamma(\alpha)} \int_{0}^{T}(T-s)^{\alpha-1} f(s, y(s)) d s \ominus \frac{-1}{\Gamma(\alpha)} \int_{0}^{x}(x-s)^{\alpha-1} f(s, y(s)) d s, x \in[0, T] .
\end{aligned}
$$

Proof. The proof is similar to Theorem 2.6, so it is omitted. 


\section{MAIN RESULTS}

Theorem 3.1. Suppose for any $z_{1}, z_{2}$, the fuzzy-valued function $f$ in problem (1) satisfies the following Krasnoselskii-Krein type conditions

(H1) $d\left(f\left(x, z_{1}\right), f\left(x, z_{2}\right)\right) \leq \frac{K \eta \Gamma(\alpha) d\left(z_{1}, z_{2}\right)}{T^{\alpha}}$, for $x \neq 0, K \eta \leq \alpha(1-\lambda), K>1$;

(H2) $d\left(f\left(x, z_{1}\right), f\left(x, z_{2}\right)\right) \leq \beta\left[d\left(z_{1}, z_{2}\right)\right]^{\gamma}, 0<\gamma<1, K(1-\gamma)<1$, and $\beta>0$ is a constant.

Then, the following successive approximations given by

$$
\begin{aligned}
& y_{n+1}(x) \\
= & \frac{\lambda}{(1-\lambda) \Gamma(\alpha)} \int_{0}^{T}(T-s)^{\alpha-1} f\left(s, y_{n}(s)\right) d s+\frac{1}{\Gamma(\alpha)} \int_{0}^{x}(x-s)^{\alpha-1} f\left(s, y_{n}(s)\right) d s,
\end{aligned}
$$

for case ${ }^{C}[(i)-g H]$ differentiability, and

$$
\begin{aligned}
& \tilde{y}_{n+1}(x) \\
= & \frac{\lambda}{(1-\lambda) \Gamma(\alpha)} \int_{0}^{T}(T-s)^{\alpha-1} f\left(s, \tilde{y}_{n}(s)\right) d s \ominus \frac{-1}{\Gamma(\alpha)} \int_{0}^{x}(x-s)^{\alpha-1} f\left(s, \tilde{y}_{n}(s)\right) d s,
\end{aligned}
$$

for case ${ }^{C}[(i i)-g H]$ differentiability, converge uniformly to two unique solutions $y(x)$ and $\tilde{y}(x)$ of problem (1) on $[0, \tilde{T}]$ respectively, where $\tilde{T}=\min \left\{T,\left(\frac{b(\Gamma(1+\alpha))}{M}\right)^{\frac{1}{\alpha}}\right\} . M$ being the bound of $f$ on $E_{0}$, i.e., $d(f, 0) \leq M$.

Proof. We prove for case ${ }^{C}[(i)-g H]$ differentiability. The successive approximations $\left\{y_{n}(x)\right\}, n=0,1, \cdots$. given by $(7)$ is well defined and continuous on $[0, \tilde{T}]$ where $\tilde{T}=$ $\min \left\{T,\left(\frac{b(\Gamma(1+\alpha))}{M}\right)^{\frac{1}{\alpha}}\right\}$. Now, for $n=1,2, \ldots$, by induction, we have

$$
\begin{aligned}
d\left(y_{n}(x), \tilde{0}\right) \leq & \frac{\lambda}{(1-\lambda) \Gamma(\alpha)} \int_{0}^{T}(T-s)^{\alpha-1} f\left(s, y_{n-1}(s)\right) d s \\
& +\frac{1}{\Gamma(\alpha)} \int_{0}^{x}(x-s)^{\alpha-1} f\left(s, y_{n-1}(s)\right) d s \\
\leq & \frac{1}{1-\lambda} \cdot \frac{M T^{\alpha}}{\Gamma(\alpha+1)} \leq \frac{b}{1-\lambda} .
\end{aligned}
$$

Note that $(7)$ is equivalent to

$$
{ }_{g H}^{C} D_{*}^{\alpha} y_{n+1}(x)=f\left(x, y_{n}(x)\right), y_{n}(0)=\lambda y_{n}(T) .
$$

So we need to prove that

$$
\lim _{n \rightarrow \infty} d\left({ }_{g H}^{C} D_{*}^{\alpha} y_{n+1}(x), f\left(x, y_{n}(x)\right)\right)=0 .
$$

In view from condition(H2), we have $K(1-\gamma)<1,0<\gamma<1$, i.e. $K<1+\gamma \sum_{i=0}^{\infty} \gamma^{i}$, and there exists an integer $N>1$ such that $K<1+\gamma \sum_{i=0}^{N-1} \gamma^{i}$. According to (9) and our assumption $d(f(x, y(x))) \leq M$ on $E_{0}$, we get

$$
d\left({ }_{g H}^{C} D_{*}^{\alpha} y_{1}(x), f\left(x, y_{1}(x)\right)\right)=d\left(f\left(x, y_{0}(x)\right), f\left(x, y_{1}(x)\right)\right) \leq 2 M,
$$


so, for $n=1,2, \cdots$,

$$
d\left({ }_{g H}^{C} D_{*}^{\alpha} y_{n}(x), f\left(x, y_{n}(x)\right)\right)=d\left(f\left(x, y_{n}(x)\right), f\left(x, y_{n-1}(x)\right)\right) \leq 2 M .
$$

From (7), (9) and (H2), we have

$$
\begin{aligned}
d\left({ }_{g H}^{C} D_{*}^{\alpha} y_{i+1}(x), f\left(x, y_{i+1}(x)\right)\right)= & d\left(f\left(x, y_{i}(x)\right), f\left(x, y_{i+1}(x)\right)\right) \leq \beta\left[d\left(y_{i}(x), y_{i+1}(x)\right)\right]^{\gamma} \\
\leq & \beta\left[\frac{\lambda}{(1-\lambda) \Gamma(\alpha)} \int_{0}^{T}(T-s)^{\alpha-1} d\left({ }_{g H}^{C} D_{*}^{\alpha} y_{i}(s), f\left(s, y_{i}(s)\right)\right) d s\right. \\
& \left.+\frac{1}{\Gamma(\alpha)} \int_{0}^{x}(x-s)^{\alpha-1} d\left({ }_{g H}^{C} D_{*}^{\alpha} y_{i}(s), f\left(s, y_{i}(s)\right)\right) d s\right]^{\gamma} .
\end{aligned}
$$

By induction, we find that

$$
d\left({ }_{g H}^{C} D_{*}^{\alpha} y_{n+1}(x), f\left(x, y_{n+1}(x)\right)\right) \leq R(T)^{\rho},
$$

where $\rho=\alpha q, q=\Sigma_{i=0}^{N-1} \gamma^{i}, N>1$ such that $K<1+q$ and

$$
R=\left(\beta^{1+\gamma+\gamma^{2}+\ldots+\gamma^{N-1}}\right)(2 M)^{\gamma^{N}}\left(\frac{1}{(1-\lambda) \Gamma(\alpha+1)}\right)^{\gamma+\gamma^{2}+\ldots+\gamma^{N}} .
$$

Well, in view of $(\mathrm{H} 1)$, for $j=0,1, \ldots, x \in(0, T]$, we obtain

$$
\begin{aligned}
& d\left({ }_{g H}^{C} D_{*}^{\alpha} y_{N+j+1}(x), f\left(x, y_{N+j+1}(x)\right)\right) \\
\leq & \frac{K \eta \Gamma(\alpha)}{T^{\alpha}} d\left(y_{N+j+1}(x), y_{N+j}(x)\right) \\
\leq & \frac{K \eta \Gamma(\alpha)}{T^{\alpha}} \frac{\lambda}{(1-\lambda) \Gamma(\alpha)} \int_{0}^{T}(T-s)^{\alpha-1} d\left({ }_{g H}^{C} D_{*}^{\alpha} y_{N+j+1}(s), f\left(s, y_{N+j}(s)\right)\right) d s \\
& +\frac{1}{\Gamma(\alpha)} \int_{0}^{x}(x-s)^{\alpha-1} d\left({ }_{g H}^{C} D_{*}^{\alpha} y_{N+j+1}(s), f\left(s, y_{N+j}(s)\right)\right) d s .
\end{aligned}
$$

From (12), we get for $j=0,1, \ldots$ and $x \in[0, T]$,

$$
d\left({ }_{g H}^{C} D_{*}^{\alpha} y_{N+j+1}(x), f\left(x, y_{N+j+1}(x)\right)\right) \leq\left(\frac{K \eta}{\alpha(1-\lambda)}\right)^{j} R T^{\rho} \leq R \mu^{j} T^{\rho} .
$$

Due to $\frac{K \eta}{\alpha(1-\lambda)}<1$, which is $\mu<1$, then the claim (10) is proved, where the constants $R, \rho, N$ are determined by $(13),{ }_{g H}^{C} D_{*}^{\alpha} y_{n}(x)$ is continuous on $[0, T]$.

Next, we prove that the sequence $y_{n}(x)$ converge uniformly on $[0, T]$, by $(14)$, it is easy to find that there is a continuous function $\varphi_{N+j+1}(x)$ on $[0, T]$, for any $j=0,1, \ldots$, such that

$$
{ }_{g H}^{C} D_{*}^{\alpha} y_{N+j+1}(x)=f\left(x, y_{N+j+1}(x)\right)+\varphi_{N+j+1}(x),
$$

where $d\left(\varphi_{N+j+1}(x), 0\right) \leq R \mu^{j} T^{\rho}$. From $(7)$,

$$
\begin{aligned}
y_{N+j+1}(x)= & \frac{\lambda}{(1-\lambda) \Gamma(\alpha)} \int_{0}^{T}(T-s)^{\alpha-1}\left[f\left(s, y_{N+j+1}(s)\right)+\varphi_{N+j+1}(s)\right] d s \\
& +\frac{1}{\Gamma(\alpha)} \int_{0}^{x}(x-s)^{\alpha-1}\left[f\left(s, y_{N+j+1}(s)\right)+\varphi_{N+j+1}(s)\right] d s .
\end{aligned}
$$


Setting $\triangle=d\left({ }_{g H}^{C} D_{*}^{\alpha} y_{N+j+1}(x),{ }_{g H}^{C} D_{*}^{\alpha} y_{N+i+1}(x)\right)$, then

$$
\begin{aligned}
& d\left(y_{N+j+1}(x), y_{N+j+1}(x)\right) \\
\leq & \frac{\lambda}{(1-\lambda) \Gamma(\alpha)} \int_{0}^{T}(T-s)^{\alpha-1} d\left(f\left(s, y_{N+j}(s)\right), f\left(s, y_{N+i}(s)\right)\right)+d\left(\varphi_{N+j+1}(s), \varphi_{N+i+1}(s)\right) d s \\
+ & \frac{1}{\Gamma(\alpha)} \int_{0}^{x}(x-s)^{\alpha-1} d\left(f\left(s, y_{N+j}(s)\right), f\left(s, y_{N+i}(s)\right)\right)+d\left(\varphi_{N+j+1}(s), \varphi_{N+i+1}(s)\right) d s .
\end{aligned}
$$

By (15), (16) and (H2), we conclude that

$$
\triangle \leq \beta\left[d\left(y_{N+j+1}(x), y_{N+j+1}(x)\right)\right]^{\gamma}+R\left(\mu^{i}+\mu^{j}\right) T^{\rho} .
$$

By (17), $\alpha \gamma<\alpha q$ and $\left(\mu^{i}+\mu^{j}\right) \leq 2$, we get

$$
\triangle \leq \beta\left(\frac{2 M+2 R T^{\rho}}{\Gamma(\alpha+1)(1-\lambda)}\right)^{\gamma} \cdot T^{\alpha q}+2 R T^{\rho} .
$$

Furthermore, due to $\rho=\alpha q$, we have

$$
\triangle \leq \beta\left[\left(\frac{2 M+2 R T^{\rho}}{\Gamma(\alpha+1)(1-\lambda)}\right)^{\gamma}+2 R\right] \cdot T^{\alpha q} \equiv R_{1} .
$$

Utilizing formula (18), we get a new estimate as follows

$$
\triangle \leq \beta\left[\left(\frac{R_{1}+2 R T^{\rho}}{\Gamma(\alpha+1)(1-\lambda)}\right)^{\gamma}+2 R\right] \cdot T^{\rho} \equiv R_{2} T^{\rho},
$$

where $R_{2}=\beta\left[\left(\frac{R_{1}+2 R T^{\rho}}{\Gamma(\alpha+1)(1-\lambda)}\right)^{\gamma}+2 R\right]$. Repeat the above process $N-1$ times, for $i, j=$ $0,1, \ldots$,

$$
\triangle \leq R_{N-1} T^{\rho}
$$

Now by using the condition (H1), we have

$$
\begin{aligned}
\triangle & \leq d\left(f\left(x, y_{N+j+1}(x)\right), f\left(x, y_{N+i+1}(x)\right)\right)+R\left(\mu^{i}+\mu^{j}\right) T^{\rho} \\
& \leq \frac{K \eta \Gamma(\alpha)}{T^{\alpha}(1-\lambda)} d\left(y_{N+j+1}(x), y_{N+i+1}(x)\right)+R\left(\mu^{i}+\mu^{j}\right) T^{\rho}
\end{aligned}
$$

which means that

$$
\triangle \leq\left[\frac{K \eta}{\alpha(1-\lambda)}\left(R_{N-1}+2 R T^{\rho}\right)+R\left(\mu^{i}+\mu^{j}\right)\right] T^{\rho} .
$$

Let $R^{*}=R_{N-1}+2 R T^{\rho}$, that is $\triangle \leq\left[\mu R^{*}+R\left(\mu^{i}+\mu^{j}\right)\right] T^{\rho}$. Then We have concluded

$$
\begin{aligned}
\triangle & \leq\left[\mu^{m-1} R^{*}+R\left(\mu^{i}+\mu^{j}\right)\left(\mu^{m-1}+\mu^{m-2}+\ldots+1\right)\right] T^{\alpha} \\
& \leq\left[\mu^{m-1} R^{*}+\left(\mu^{i}+\mu^{j}\right) \frac{R}{1-\mu}\right] T^{\alpha} .
\end{aligned}
$$

Therefore, by $\mu<1$, we get $\triangle \rightarrow 0$ as $i, j, m \rightarrow \infty$. Thus the sequences $f\left(x, y_{n}(x)\right)$ satisfies the Cauchy criterion, that is the sequences $f\left(x, y_{n}(x)\right)$ is uniformly convergent on $x \in[0, T]$, and the sequence $y_{n}$ is also uniformly convergent on $[0, T]$.

Let $y(x)$ be the limit function of $y_{n}(x)$, it is easy to see that $y(x)$ is the solution of problem (1) in the sense of ${ }^{C}[(i)-g H]$ differentiability. If it's not true, we will consider 
$y_{1}(x)$ and $y_{2}(x)$ are the solution of problem (1) if $y_{1}, y_{2}$ is ${ }^{C}[(i)-g H]$ differentiable. Let $\phi(x)=d\left(y_{1}(x), y_{2}(x)\right)$, by $(3)$ and $(\mathrm{H} 1)$, we obtain

$$
\begin{aligned}
\phi(x) \leq & \frac{\lambda}{(1-\lambda) \Gamma(\alpha)} \int_{0}^{T}(T-s)^{\alpha-1} d\left(f\left(s, y_{1}(s)\right), f\left(s, y_{2}(s)\right)\right) d s \\
& +\frac{1}{\Gamma(\alpha)} \int_{0}^{x}(x-s)^{\alpha-1} d\left(f\left(s, y_{1}(s)\right), f\left(s, y_{2}(s)\right)\right) d s \\
\leq & \frac{\lambda k \eta}{(1-\lambda) \Gamma(\alpha)} \int_{0}^{T}(T-s)^{\alpha-1} \frac{\phi(s)}{T^{\alpha}} d s+k \eta \int_{0}^{x}(x-s)^{\alpha-1} \frac{\phi(s)}{T^{\alpha}} d s .
\end{aligned}
$$

Next, we need to prove uniqueness. Let $\psi(x)=\frac{\phi(x)}{T^{\alpha}}$, we only prove that $\psi(x) \equiv 0$, that is $\phi(x) \equiv 0$. In fact, let $0<h=\max _{[0, \tilde{T}]} \psi(x)=\psi\left(x_{1}\right)$, then we have

$$
\begin{aligned}
h=\psi\left(x_{1}\right) & \leq \frac{\lambda k \eta h}{(1-\lambda) T^{\alpha}} \int_{0}^{T}(T-s)^{\alpha-1} d s+\frac{k \eta h}{T^{\alpha}} \int_{0}^{x_{1}}\left(x_{1}-s\right)^{\alpha-1} d s \\
& \leq \frac{k \eta h}{(1-\lambda) \alpha} \leq h,
\end{aligned}
$$

which is a contradiction due to $k \eta \leq \alpha(1-\lambda)$. Therefore, we show that uniqueness of solution of FFDES (1) in the sense of ${ }^{C}[(i)-g H]$ differentiability.

For case ${ }^{C}[(i i)-g H]$ differentiability is completely similar to previous one, hence is omitted. The proof is complete.

Acknowledgements. The authors would like to express sincere thanks to the anonymous referee for his/her carefully reading the manuscript and valuable comments and suggestions. This work is supported by Natural Science Foundation of Gansu Province (No. 20JR5RA522), Science Research Project for Colleges and Universities of Gansu Province (No. 2019B-047), Project of NWNU-LKQN2019-13 and Doctoral Research Fund of Northwest Normal University (No. 6014/0002020209).

\section{REFERENCES}

[1] P. Chen, X. Zhang, Y. Li. Fractional non-autonomous evolution equation with nonlocal conditions. J. Pseud0-Differ. Oper., 10:955-973, 2019.

[2] P. Chen, X. Zhang, Y. Li. Cauchy problem for fractional non-autonomous evolution equations. Banach. J. Math. Anal., 14:559-584, 2020.

[3] P. Chen, X. Zhang, Y. Li. Existence and approximate controllability of fractional evolution equations with nonlocal conditions via resolvent operators. Fract. Cal. Appl. Anal., 23:268-291, 2020.

[4] P. Chen, X. Zhang, Y. Li. Approximate controllability of non-autonomous evolution system with nonlocal conditions. J. Dyn. Control. Syst., 26:1-16, 2020.

[5] L.A. Zadeh. Fuzzy Sets. Infor. Control., 8:338-353, 1965.

[6] O. Kaleva. Fuzzy Differential Equations. Fuzzy. Set. Syst., 24:301-317, 1987.

[7] M. Puri, D. Ralsecu. Differentials of Fuzzy Functions. J. Math. Anal. Appl., 91:552-558, 1983. 
[8] D.N. Georgiou, J.J. Nieto, R. Rodrigurz-Lopez. Initial Value Problems for Higher-Order Fuzzy Differential Equations. Nonlinear. Anal-Theor., 63: 587-600, 2005.

[9] Z.T. Gong, Y.B. Shao. Global Existence and Uniqueness of Solutions for Fuzzy Differential Equations Under Dissipative-Type Conditions. Comput. Math. Appl., 56: 2716-2723, 2008.

[10] S.J. Song, C.X. Wu, X.P. Xue. Existence and Uniqueness of Cauchy Problem for Fuzzy Differential Equations under Dissipative Conditions. Comput. Math. Appl., 51:1483-1492, 2006.

[11] S.J. Song, L. Guo, C.B. Feng. Global Existence of Solutions to Fuzzy Differential Equations. Fuzzy. Set. Syst., 115:371-376, 2000.

[12] J.P. Aubin. Fuzzy Differential Inclusions. Problems. Control. Inform. Theor., 19:55-67, 1990.

[13] A. Khastan, R. Rodríguez-López. On Periodic Solutions to First Order Linear Fuzzy Differential Equations Under Differential Inclusion's approach. Inform Sciences., 322:31-50, 2015.

[14] B. Bede, S. G. Gal. Generalizations of the Differentiability of Fuzzy-Number-Valued Functions with Applications to Fuzzy Differential Equations. Fuzzy. Set. Syst., 151:581-599, 2005.

[15] B. Bede, L.A. Stefanini. Generalized Differentiability of Fuzzy-Valued Functions. Fuzzy. Set. Syst., 230:119-141, 2013.

[16] L.A. Stefanini. A Generalization of Hukuhara Difference and Division for Interval and Fuzzy Arithmetic. Fuzzy. Set. Syst., 161:1564-1584, 2010.

[17] A. Khastan, J.J. Nieto, R. Rodríguez-López. Fuzzy Delay Differential Equations under generalized Differentiability. Inform Sciences., 2014, 275: 145-167.

[18] N.V. Hoa, N,D, Phu. Fuzzy Functional Integro-Differential Equations Under Generalized HDifferentiability. J. Intell. Fuzzy. Syst., 26:2073-2085, 2014.

[19] T. Allahviranloo, L. Hooshangian. Fuzzy Generalized H-Differential and Applications to Fuzzy Differential Equations of Second-Order. J. Intell. Fuzzy. Syst., 26:1951-1967, 2014.

[20] H.V. Ngo, V. Lupulescu, D.O. Regan. A Note On Initial Value Problems for Fractional Fuzzy Differential Equations. Fuzzy. Set. Syst., 347:54-69, 2018.

[21] A.V. Truong, V. Ho, H.V. Ngo. The Existence of Solutions for an Initial Value Problem of CaputoHadamard-type Fuzzy Fractional Differential Equations of Order $\alpha \in(1,2)$. J. Intell. Fuzzy. Syst., 36:5821-5834, 2019.

[22] H. V. Ngo. On the Initial Value Problem for Fuzzy Differential Equations of Non-Integer Order $\alpha \in(1,2)$. Soft Computing., 24:935-954, 2020.

[23] E.J. Villamizar-Roa, V. Angulo-Castillo, Y. Chalco-Cano. Existence of Solutions to Fuzzy Differential Equations with Generalized Hukuhara Derivative via Contractive-Like Mapping Principles. Fuzzy. Set. Syst., 265:24-38, 2015.

[24] R. Alikhani, F. Bahrami. Global Solutions of Fuzzy Intergro-Differential Equations Under Generalized Differentiability by The Method of Upper and Lower Solutions. Inform Sciences., 295:600608, 2015.

[25] M.L. Puri, D. Ralescu. Fuzzy Random Variables. J. Math. Anal. Appl., 114:409-422, 1986.

[26] B. Bede, L. Stefanini. Generalized Differentiability of Fuzzy-Valued Functions. Fuzzy. Set. Syst., 2012.

[27] T. Allahviranloo, A. Armand, Z. Gouyandeh. Fuzzy Fractional Differential Equations under Generalized Fuzzy Caputo Derivative. J. Intell. Fuzzy. Syst., 26:1481-1490, 2014.

[28] T. Allahviranloo, S. Salahshour, S. Abbasbandy. Explicit solutions of fractional differential equations with uncertainty. Soft Computing., 297-302, 2012.

[29] I. Podlubny. Fractional Differential Equations. Academic Press, San Diego, 1999.

[30] S. Salahshour, T. Allahviranloo, S. Abbasbandy, D. Baleanu. Existence and Uniqueness Results for Fractional Differential Equations with Uncertainly. Adv. Differ. Equ-ny, 2012: 112, 2012. 